\title{
Connected Diagnostics to improve accurate diagnosis, treatment, and conditional payment of malaria services in Kenya.
}

Shannen Van Duijn ( $\nabla$ s.vanduijn@pharmaccess.org )

PharmAccess Foundation https://orcid.org/0000-0003-1361-2711

Angela Siteyi

PharmAccess Foundation Kenya

Sherzel Smith

PharmAccess Foundation

Emmanuel Milimo

PharmAccess Foundation Kenya

Leon Stijvers

PharmAccess Foundation

Monica Oguttu

Kenyan Medical and Educational Trust

Michael Amollo

Nigthingale Hospital

Edward Okeyo

PharmAccess Foundation Kenya

Lilyana Dayo

Malaria Control Program, Ministry of Health

Titus Kwambai

Kenya Medical Research Institute

Dickens Onyango

County Department of Health Kisumu

Tobias Rinke de Wit

PharmAccess Foundation and Joep Lange Institute

Research article

Keywords: Diagnosis, Treatment, Conditional payments, Malaria, Kenya, Connected Diagnostics

Posted Date: November 20th, 2020

DOl: https://doi.org/10.21203/rs.3.rs-36894/v2 
License: (c) (i) This work is licensed under a Creative Commons Attribution 4.0 International License. Read Full License

Version of Record: A version of this preprint was published at BMC Medical Informatics and Decision Making on August 4th, 2021. See the published version at https://doi.org/10.1186/s12911-021-01600-z. 


\section{Manuscript Research article} BMC Medical Informatics and Decision Making

\section{TITLE}

3 Connected Diagnostics to improve accurate diagnosis, treatment, and conditional payment of

4 malaria services in Kenya.

\section{AUTHORS}

6 Shannen M.C. van Duijn ${ }^{1}$, Angela .K. Siteyi ${ }^{2}$, Sherzel. Smith ${ }^{1}$, Emmanuel Milimo ${ }^{2}$, Leon Stijvers ${ }^{1}$, Monica

7 Oguttu $^{3}$, Michael O. Amollo ${ }^{4}$, Edward O. Okeyo ${ }^{2}$, Lilyana Dayo ${ }^{5}$, Titus Kwambai ${ }^{6}$, Dickens Onyango ${ }^{7}$,

8 Tobias F. Rinke de Wit ${ }^{1,8}$

\section{Author's affiliation}

$10{ }^{1}$ PharmAccess Foundation, Amsterdam, The Netherlands

$11 \quad{ }^{2}$ PharmAccess Foundation, Nairobi, Kenya

$12 \quad{ }^{3}$ Kisumu Medical \& Education Trust (KMET), Kenya

$13{ }^{4}$ Nightingale Hospital, Kisumu, Kenya

$14{ }^{5}$ Malaria Control Program Coordinator-Kisumu County - Ministry of Health, Kenya

$15 \quad{ }^{6}$ Kenyan Medical Research Institute (KEMRI), Kisumu, Kenya

$16{ }^{7}$ Country Director, Medical Services Kisumu County, Kenya

$17{ }^{8}$ Joep Lange Institute, Amsterdam, The Netherlands

18 Corresponding Author: Shannen van Duijn (s.vanduijn@ pharmaccess.org) 


\section{ABSTRACT}

24 Background: In sub-Saharan Africa, the material and human capacity to diagnose patients reporting with

25 fever to healthcare providers is largely insufficient. Febrile patients are typically treated presumptively with

26 antimalarials and/or antibiotics. Such over-prescription can lead to drug resistance and involves

27 unnecessary costs to the health system. International funding for malaria is decreasing and transition to

28 domestic funding is challenged by UHC efforts and recent COVID-19 outbreak. Herewith we present a

29 digital approach to markedly improve efficiencies in diagnosis and treatment of malaria in endemic Kisumu,

30 Kenya. The objective of this study is to evaluate feasibility, user experience, clinical performance and of

31 Connected Diagnostics in Kisumu and to assess over-prescription of antimalarials.

32

33 Methods: Our intervention was performed Oct 2017 - Dec 2018 across seven providers in Kisumu. Patients

34 were enrolled on M-TIBA platform, diagnostic test results digitized, and only positive patients were

35 digitally entitled for malaria treatment. Data on socio-demographics, healthcare transactions and medical

36 outcomes were analysed using standard descriptive quantitative statistics. Provider perspectives were

37 gathered by 19 semi-structured interviews.

39 Results: In total 11,689 febrile patients were tested. Malaria positivity rates ranged from $7.4 \%$ to $30.2 \%$

40 between providers, with significantly more positive cases amongst the poor $(\mathrm{p}<0.05)$. Over-prescription of

41 antimalarials was $28 \%$, fluctuating between $4.6 \%$ to $63.3 \%$ per provider. Prescription of branded versus

42 generic antimalarials was dichotomous. Challenges were encountered transitioning from microscopy to

43 RDT.

44

45 Conclusion: We provide full proof-of-concept of innovative Connected Diagnostics to use digitized 46 malaria diagnostics to earmark digital entitlements for correct malaria treatment of patients. This approach

47 has large cost-saving and quality improving potential. 
49 Keywords: Diagnosis, Treatment, Conditional payments, Malaria, Kenya, Connected Diagnostics

\section{MANUSCRIPT}

\section{Background}

52 Sub-Saharan Africa (SSA) continues to face challenges attaining accurate diagnosis of both infectious and

53 non-communicable illnesses. Laboratory diagnosis remains suboptimal with much-needed lab equipment

54 often lacking, financial resources being scarce and skilled medical staff underrepresented [1]. Recently

55 across 49 SSA countries only 380 laboratories were found to be accredited against international standards,

56 with many countries not even hosting one single accredited provider [2]. Also in urban and semi-urban

57 centers where lab facilities and staff are usually better available, inaccurate diagnoses with limited

58 sensitivity and specificity are common practice [1, 3-5]. Fever is one of the most common reasons people

59 in Africa visit health providers. However, due to the aforementioned challenges with lab providers across

60 the continent, febrile patients are often not diagnosed using laboratory tests, but only presumptively, based

61 on clinical grounds [1]. The most common presumptive diagnosis in febrile patients is malaria, followed

62 by bacterial infection(s), resulting in over-prescription of antimalarials and antibiotics. A recent study

63 indicated that more than $70 \%$ of fever cases in Tanzanian children was caused by viral infections, against

64 which antimalarials and antibiotics do not work [6].

65

66 SSA countries hold a disproportionately high share of global malaria burden. In 2018, the region hosted

$6793 \%$ of malaria cases and $94 \%$ of malaria deaths [7]. Over $99 \%$ of malaria cases in malaria endemic areas

68 of Africa were caused by P. falciparum in 2018 [7]. The malaria morbidity and mortality in Africa cost the

69 continent more than 12 billion USD annually in GDP [8, 9]. Furthermore, global funding for malaria control

70 and elimination in Africa is flat-lining and the recent call for universal health coverage (UHC) puts pressure 
71 on traditional vertical funds for malaria, such as the Global Fund to Fight Aids Tuberculosis and Malaria

72 (GFATM) and President's Malaria Initiative (PMI) [10, 11]. Additional challenges to malaria service

73 delivery are emerging with the recent outbreak of COVID-19 [12]. Important drawbacks in combatting

74 malaria were encountered in West Africa during the recent Ebola outbreak, setting examples for responding

75 to the imminent COVID-19 pandemic [13], indicating importance of more efficient, targeted digital malaria

76 service delivery.

77

78 Because of presumptive diagnosis and treatment for malaria in febrile patients, over-prescription of malaria

79 drugs in malaria endemic African countries is common [14, 15]. This can have key health implications,

80 including development of drug resistance, higher risk of treatment failure, increased morbidity. In addition,

81 it leads to economic implications such as unnecessary drug costs, recurring visits, and economic

82 productivity losses due to longer sick days. Due to this, the costs for both households and healthcare

83 providers increases $[16,17]$. Moreover, prescription of drugs that are ineffective can lead to reduced patient

84 trust in healthcare provision with subsequent decreased willingness to participate in financial (insurance)

85 schemes for pre-payment and risk-sharing.

86

87 Recent developments in the area of digital technology provide new opportunities to address the above

88 situation in a radically different way. Firstly, the emergence of the Internet of Things (IoT) comprising a

89 rapidly growing arsenal of 'digital diagnostics': tools and devices that digitalize and link human physical

90 parameters to the internet, complete with sensing and measuring capabilities [18]. Secondly, the mobile

91 revolution: $75 \%$ of the African population has access to a mobile phone. In Kenya, mobile penetration is at

$9286 \%$, and still growing rapidly $[19,20]$. Thirdly, Africa is leapfrogging with the advent of 'bankless

93 banking': digital payment systems through mobile phones transferring entitlements between individuals.

94 An example of this is M-PESA, launched in 2007 in Kenya: an electronic mobile money service to store,

95 send and receive money on any mobile phone (smartphone or non-feature) with an M-PESA account [21]. 
97 Since 2014 the non-profit foundation PharmAccess has leveraged these developments to create a 'mobile

98 health wallet' linked to the M-PESA payment platform, known as M-TIBA ('mobile therapy' in Swahili).

99 Today, M-TIBA is hosted by CarePay International and represents the first African digital platform

100 exchanging data and funds/entitlements that are exclusively earmarked for health and healthcare [22]. M-

101 TIBA works on simple, non-feature mobile phones as well as smartphones. It connects patients, healthcare

102 providers and healthcare payers (such as insurers and donors) and exchanges data and entitlements between

103 them. Users can save into their own (family) wallets, they can receive money from relatives elsewhere in

104 the country, from donors, and even from individuals in other countries willing to donate directly for health

105 [23]. This is a first step in creating new digital solidarity mechanisms where people can financially

106 contribute to each other's health: the rich for the poor, the healthy for the sick, the young for the old, and

107 communities for individuals. As of April 2020, 4.2 million people in Kenya, Nigeria, Tanzania and 1,500

108 health providers on the continent were connected to the platform [22].

109

110 Given above developments, we saw an opportunity within the Kenyan context to combine digital 111 diagnostics and M-TIBA technology to support marked improvements in the efficiency of diagnosis and 112 treatment of febrile diseases. This approach is referred to as 'Connected Diagnostics (ConnDx)'. ConnDx

113 introduces a new system-wide digital delivery approach for malaria care, linking phones to diagnostics and

114 payment systems. Providing ConnDx services, yields simultaneous real-time insight into data and funds

115 exchanged between patients, providers, and healthcare payers.

116

117 ConnDx digitalizes rapid diagnostic tests (RDTs) through photography and interprets and stores results in 118 cloud-based databases through dedicated RDT readers. Such readers are available, like the Fio Deki 119 Reader ${ }^{\mathrm{TM}}$, i-CalQ lateral flow-test imaging ${ }^{2}$ and the Mobile Assay 'lab-on-mobile device' platform ${ }^{3}$. The

\footnotetext{
${ }^{1}$ http://fio.com/rapid-testing/

2 https://i-calq.com/technology/

${ }^{3}$ https://mobileassay.com/
} 
120 digital diagnostic results subsequently inform a mobile phone-based payment system (M-TIBA) to channel

121 dedicated funds for diagnosis and treatment of the pertinent disease to the provider and/or patient. A recent

122 proof-of-principle of ConnDx, was provided in Samburu, Kenya, in which RDTs interpreted by the Fio

123 Deki Reader ${ }^{\mathrm{TM}}$ were used to diagnose brucellosis in remote populations [24]. Brucellosis, a rare bacterial

124 zoonotic illness, is often underdiagnosed due to having similar febrile symptoms as malaria [25]. Patients

125 testing positive were directly linked to funds through their respective mobile health wallets to allow for

126 payment of drugs for the pertinent diseases, conditional to positive diagnosis of these diseases. Feasibility

127 was demonstrated, patients were correctly treated, their user experience was positive, and hotspots of

128 brucellosis were identified [24].

129

130 Having proven that ConnDx could be effective in identifying a rare disease in rural, remote populations,

131 this paper reports on the next step: scaling ConnDx to a more densely populated area for a more prevalent

132 disease (malaria) that causes high morbidity, mortality and serious economic impact. The objective of this

133 study is to evaluate feasibility, user experience, and clinical performance of a ConnDx intervention in

134 Kisumu, Kenya. Additionally, the aim is to assess over-prescription of antimalarials in this population. The

135 location of choice, Kisumu County in Kenya, has a population of 1.2 million [26], and a reported high

136 malaria prevalence of approximately $28-35 \%$ in the general population [27, 28]. Our intervention took place

137 from October 2017 - December 2018 amongst seven private sector health providers and $\sim 12,000$

138 beneficiaries.

\section{Methods}

140 The ConnDx process starts upon patient presentation at participating providers or healthcare outreach

141 events. The project was announced to the public through posters in the waiting room referring to a free

142 'Malaria Test \& Treat Campaign'. Consenting patients presenting with fever and/or malaria symptoms were 
143 referred by informed clinicians for malaria testing by qualified lab technicians using malaria RDTs,

144 digitalized using a Fio Deki Reader ${ }^{\mathrm{TM}}$. The Reader contained a drop-down menu to collect demographic

145 data (gender, age, pregnancy status), basic economic data (socio-economic status) and geographic location

146 data (community of residence, to a granularity level of 3,000-5,000 citizens). For socio-economic status

147 assessment three questions were added to the menu pertinent to access to electricity, toilet type and

148 education level of household head. These questions had been previously identified most informative by

149 principal component analysis using a Multiple Indicator Cluster Survey in Nyanza for reference [29]. This

150 led to a poverty index, in which level 1 corresponds to the poorest category; level 3 to the least poor.

152 Simultaneously, the participating patients were enrolled on the M-TIBA platform and individual health

153 wallets were created. The collected Fio Deki Reader ${ }^{\mathrm{TM}}$ demographic data, along with patient test results,

154 was uploaded to the Fionet cloud database and linked to the patient's mobile health wallet account using a

155 unique M-TIBA transaction code. Only patients with a positive diagnosis were entitled to receive funding

156 for antimalarials, which (in an interim functionality) was paid to their respective healthcare providers, thus

157 effectuating fully paid treatment for the patients. Figure 1 provides a schematic illustration of the ConnDx 158 process.

160 ConnDx was implemented from October 2017 - December 2018 across seven private care providers

161 (anonymized A-G) within sub-counties of Kisumu County. The providers were a part of an existing care

162 system. They were selected based on (high) patient throughput and geographic location and ranged from

163 smaller providers with set opening hours to large 24/7 hospital facilities. The National District Health

164 Information System (DHIS-2) software was used as data source for selection of providers.

166 The provider perspectives on the use of ConnDx were gathered by means of 19 semi-structured interviews 167 in the seven participating health providers. Interviewed staff were managers, lab technicians, receptionists, 
and doctors or nurses. The interviews were analyzed by applying a thematic analysis approach using the

169 software Atlas.ti to identify patterns or topics within the data.

170

171 Quantitative data from both cloud-based databases (FIO and M-TIBA) were analyzed through descriptive

172 statistics in Stata 12 and Microsoft Excel 2016. Descriptive analyses were done for each provider separate

173 and over time. Relationships between categorical variables were tested by using the chi-square test to

174 calculate $p$-values and compare proportions between groups. A p-value of $<0.05$ was considered statistically

175 significant. For geographic location analyses, the subdivision of Kisumu County was divided into the 163

176 administrative community units, each with roughly 1,000-1,200 households. Participants were asked at

177 point of care which community unit their home was based. Geographic analyses were performed in QGIS

178 (Version 3.0) by using the geographic center of each community unit as a proxy for the location of the

179 participants' domicile. The project was positioned as a quality improvement project of existing malaria

180 services according to Kenyan Guidelines. It was run in parallel to existing systems and services. All study

181 participants provided consent for participation prior to the enrolment process into M-TIBA. Verbal

182 informed consent was obtained from provider interviewees prior to the interview.

\section{Results}

184 Between October 2017 and December 2018, 11,689 people with fever were tested in 7 private providers 185 (A-G). Figure 2 depicts the numbers of malaria RDTs uploaded by location of these providers and 186 participants' domiciles according to the community unit they reported to live in.

188 Figure 3 shows the participation dynamics per provider during the intervention. Providers A and B 189 discontinued participation after 5 months; providers E, F and G enrolled in April 2018 and continued until 190 the end of the project. Providers A and B are not included in further analyses, due to the insufficient amount 191 of data for the study period. Malaria testing rates coincided with the rainy seasons in Kisumu for 2017 and 
1922018 ("long rains" from March to June, and "short rains" from October to December), known to increase 193 malaria transmission. During the study period (2018), rainfall in Kisumu was particularly high and 194 prolonged compared to the average [30].

196 Variation between the providers was found regarding positivity rates of malaria tests. Provider D reported 197 relatively the least positive malaria tests $(7.4 \% ; 290 / 3,908)$. This is followed by provider E $(12.6 \%$; 198 127/1,006), provider G $(16.7 \% ; 279 / 1,666)$ and provider C $(18.1 \%$; 460/2,548). Provider F identified the 199 highest percentage of positive malaria cases $(30.2 \%$; 922/3,057).

201 From all 11,689 consenting patients who were tested for malaria, there was an $18.3 \%$ overall test positivity 202 rate. Although more women were tested (58.2\%), more male positive cases $(19.0 \%$; 993/7,297) were found $203(15.4 \% ; 1,126 / 7,297)$. The mean age of patients was 23.6 years and $16.5 \%$ of patients were aged under 5 204 years old. For children under 5 years of age, the percentage of positive malaria cases was comparable to 205 adults $17.7 \%(\mathrm{n}=340 / 1,926)$. From the participants, $63.1 \%$ were member of NHIF, the national health 206 insurance in Kenya. Patients in poverty level 1 were significantly more often tested positive for malaria 207 than patients with a higher poverty score $(p$-value $<0.05)$.

209 The RDT results uploads of ConnDx allowed for quality assessment, since the Deki Reader ${ }^{\mathrm{TM}}$ indicates

210 'error' at point of care when the RDT is not performed correctly. Figures 4a-c illustrate quality improvement 211 of RDT performance represented by reductions of error rates over time. Figure 4a demonstrates 212 considerable overall improvement over the first month of RDT usage (from $25 \%$ range down to below 5\%).

213 Figures $4 \mathrm{~b}$ and $4 \mathrm{c}$ provide individual data for later starters $\mathrm{G}$ (improvement) and F (continued good 214 performance).

216 For all participating providers, general over-prescription of antimalarials was observed. Over-prescription 217 is defined by the proportion of antimalarials dispensed to the actual number of positive cases as identified 
218 by a positive malaria test. The overall over-prescription was $28.0 \%$, fluctuating between $4.6 \%$ (provider F)

219 and $63.3 \%$ (provider D). There were fluctuations in over-prescription over time, as illustrated in Figure 5.

220 High over-prescription rates were recorded prior to the introduction of ConnDx at provider D. During the

221 ConnDx intervention over-prescription declined significantly. Only towards the end of the intervention, an

222 increase was observed.

223

224 Figure 6 demonstrates M-TIBA derived information on individual provider specific prescribing behavior 225 in choices of drugs $\left(1^{\text {st }}\right.$ line and $2^{\text {nd }}$ line $)$ for antimalarials. The most common prescriptions were: 226 artemether/lumefantrine (75.4\%, either branded (Coartem, 43.7\%) or generic (28.5\%), followed by 227 artemether injection, a $2^{\text {nd }}$ line drug (15\%). Overall, $75.6 \%$ of all antimalarials dispensed by the providers 228 were $1^{\text {st }}$ line and $24.4 \%$ were $2^{\text {nd }}$ line. It was found that 2 nd line antimalarials were significantly more 229 frequently prescribed to the more affluent population and to participants who were not insured with NHIF 230 (p-value <0.05). Provider $\mathrm{C}$ dispensed most $2^{\text {nd }}$ line antimalarials (average 53.7, ranging from 26.5\% 231 100.0\%). This is followed by provider $\mathrm{F}(24.7 \%)$, provider D $(9.2 \%)$, provider $\mathrm{G}(7.1 \%)$ and provider E 232 (3.9\%). Figure 6 illustrates that three (C, E, F) out of five private providers significantly diverted from the 233 Kenyan guidelines for prescription. The choice of branded versus generic antimalarials appeared 234 dichotomous: providers $\mathrm{C}, \mathrm{D}$ and $\mathrm{G}$ prescribed predominantly generics and providers $\mathrm{E}$ and $\mathrm{F}$ branded 235 drugs.

237 Our qualitative interviews with healthcare providers identified several opportunities and challenges of 238 ConnDx. In general, positive feedbacks were received by all interviewees. Malaria diagnosis through RDT 239 was experienced by 14 of 19 providers as easier, more efficient, and faster. Interviewed providers (14/19)

240 predominantly mentioned patients were also positive towards this way of testing for malaria and receiving 241 payments for treatment. In addition, healthcare providers predominantly (13/19) appreciated ConnDx 242 providing interesting additional (management) information on patients, prescription practices and drug 243 procurement. Interviewees (16/19) indicated ConnDx to be an effective means to expand access to malaria 
244 treatment for the poor and for children. Overall, 13 of 19 providers reported an increased awareness that

245 diagnostics should determine treatment decisions, and thereby reduce the prescriptions of unnecessary 246 drugs. One of the challenges most frequently mentioned by healthcare providers (14/19) was a persistent 247 lack of trust in RDTs.

\section{Discussion}

249 This study describes feasibility, user experience and clinical performance through healthcare providers in

250 Kisumu of a novel digital approach to malaria diagnosis that directs conditional payments for malaria

251 treatment: ConnDx. We demonstrate significant potential for increasing efficiencies of malaria service

252 delivery in the Kenyan private healthcare sector with respect to better diagnosis, reducing over-prescription,

253 selecting correct $1^{\text {st }}$ and $2^{\text {nd }}$ line drug combinations and reducing malaria transaction costs, while at the

254 same time generating valuable real-time data on malaria prevalence and incidence (hotspots) that can be

255 fed into national information systems (such as DHIS-2).

257 First of all, ConnDx proved through this pilot its potency to monitoring malaria epidemiology in semi-real 258 time and generate important data for malaria management. Considerable variation was revealed between 259 providers, with malaria positivity rates ranging from $7.4 \%$ (provider D) to $30.2 \%$ (provider F). This led to 260 verifiable assumptions such as Provider D being a referral hospital and therefore less likely to serve primary 261 malaria cases, while provider F, being located near wet rice fields, serving known hotspots for malaria.

262 During months with more rainfall, there were significantly more malaria tests done at the providers ( $\mathrm{p}$-value

263 <.05). However, no significant correlation was found between months with more rainfall and positive 264 malaria test results. Providers located in or near low-income settlements ( $C$ and $G)$ appeared to have higher 265 malaria positivity rates, as a correlation between poorer patients and positive malaria tests was found (p266 value $<0.05)$. ConnDx showed a relatively low participation rate of children: only $16.5 \%$ of reported 267 patients were aged $<5$ years. Moreover, positive malaria rates in this age group were not different from 
adults: $17.7 \%$. This seems an underestimation of the cases, as children under five are at considerably higher risk of contracting malaria than adults. They are also the most vulnerable group affected by malaria, WHO 270 estimated $67 \%$ of malaria deaths globally were children $<5$ years old in 2018 [7]. Our qualitative interviews 271 with providers revealed that clients experienced challenges subscribing their children to M-TIBA as 272 dependents, therefore erroneously reporting them as adult primary members. This was corrected later during 273 the campaign but could have contributed to general underreporting of pediatric malaria cases. All in all, it 274 was clearly demonstrated that ConnDx can facilitate in semi-real time important healthcare provider275 differences in malaria case management. Such information, when collected at a larger-scale level could 276 help policy makers and health system managers to target their efforts for (human) malaria capacity building.

278 Secondly, this study demonstrated the overall potency of ConnDx to monitor provider prescription 279 behaviors and identify practices that are significantly aberrant from Kenyan National Guidelines. Important 280 overall over-prescription was recorded of antimalarials (28.0\%), varying between providers from $4.6 \%$ 281 (provider F) to $63.3 \%$ (provider D). There are multiple reasons for over-prescription, ranging from 282 monetary considerations of private providers, to patient expectation and pressure to receive drugs, 283 avoidance of clinicians to take the risk of a false negative diagnosis and subsequent fatality, etc. [15]. Our 284 qualitative interviews mostly pointed towards patient pressure (10/19 interviewees). Furthermore, ConnDx 285 revealed an unexpected and erroneously high level of prescription of $2^{\text {nd }}$ line antimalarials (overall 28.0\%). 286 Provider $\mathrm{C}$ revealed $2^{\text {nd }}$ line prescription levels of overall $53.7 \%$, at times going up to even $100 \%$. This is 287 remarkable, as $2^{\text {nd }}$ line antimalarials are generally used for severe cases of malaria, which represent on 288 average $<2 \%$ [31], or in (rare) cases of suboptimal parasitological response with $1^{\text {st }}$ line antimalarials 289 (resistance). When probed with this observation, provider $\mathrm{C}$ reported a prolonged stock-out of $1^{\text {st }}$ line 290 antimalarials and therefore switching to $2^{\text {nd }}$ line. Over-prescription of $2^{\text {nd }}$ line antimalarials was significantly 291 more frequently found with more affluent and uninsured participants. This could indicate providers are 292 aware of the socio-economic status of their clients and they incorporate this into their prescriptions. 293 Moreover, it appeared there was a very dichotomous, almost exclusive usage of either branded (provider E 
294 and F) or generic (provider C, D, G) antimalarials. This could be because providers serving more affluent 295 customers prefer procurement of branded versus generic antimalarials. Conversely, more affluent 296 customers might request for branded instead of generic antimalarials. Often, generic medicines are 297 considered to be of poor quality and treated with more suspicion than branded medicines [32, 33].

299 Third this study indicates that ConnDx can increase efficiency in malaria service delivery by decreasing 300 costs in several ways. Over-prescription of antimalarials can be monitored, aberrations identified, and 301 actions undertaken to address those. A 2013 study conducted in four providers in western Kenya, noted that 302 presumptive malaria treatment lead to misdiagnosis rates as high as $53 \%$. The same study found that as 303 many as $36 \%$ of patients diagnosed with malaria via microscopy should be classified as false positives [14].

304 ConnDx can play an important role to reduce such figure. Further cost reductions can potentially be realized 305 by ConnDx decreasing paperwork in health providers; such automated systems saving time, manpower and 306 being more accurate in reporting cases [34]. In addition, ConnDx implies less dependency on expensive 307 and maintenance-dependent microscopy, and less electricity will be required to perform diagnostics tests.

308 Moreover, due to its use-friendliness ConnDx, will provide more opportunities for lower trained lab-staff 309 to perform such tests, saving personnel costs. Finally, and most importantly, ConnDx can facilitate a much 310 more targeted bottom-up payment for malaria services to providers and clients, creating unprecedented 311 transparency as compared to current top-down systems.

313 Overall user experiences of ConnDx from the perspectives of providers were positive. Nevertheless, after 314 five months of implementation, in February 2018 two providers, A and B, dropped out of the pilot. Probed 315 through our interviews, these providers reported reluctance of their patients (who were already covered by $316 \mathrm{NHIF)}$ to go through the administrative process of registering with M-TIBA. Other, more general 317 challenges identified were technical, such as dependency of ConnDx from internet connectivity. However, 318 for all providers, the key challenge were reservations of their staff to adopt the use of RDTs instead of 319 microscopy. In general, microscopy was still seen as the golden standard for malaria testing in Kenya, 
321 healthcare providers mentioned they verified RDT results by microscope.

323 Indeed, ConnDx is dependent on the use of RDTs instead of microscopy for malaria diagnostic testing.

324 Apart from National Guidelines, also the international literature reports sensitivity and specificity of malaria

325 RDTs equal to microscopy [36, 37]. RDTs are also recommended by WHO [3]. Several studies

326 demonstrated impaired sensitivity of microscopy in actual field situations in Africa as compared to perfectly

327 controlled laboratory circumstances, with regular refresher training being required [33, 34]. RDTs have the

328 added advantage that, in contrast to microscopy, these can easily be externally quality controlled by visual

329 inspection of independent third parties. This opportunity is further enhanced through the ConnDx feature

330 of making digital photographs of every test result, stored in secure cloud-based databases that can be

331 accessed anywhere in the world. Additional advantage of RDT is that results can be digitalized, which

332 accelerates data collection to (semi)real-timeliness, allows for telemedicine-based quality control and

333 improves quality and completeness of data collection (versus paper-based malaria files being entered into

334 national DHIS-2 systems on a several-time-per-year basis). These options are all much more problematic

335 when performing microscopy. Moreover, in the sub-Saharan African reality, RDTs can readily detect

336 Plasmodium falciparum, which causes the highest malaria morbidity and mortality and represents $99.7 \%$

337 of cases [35]. RDTs indeed are less available that specifically detect $P$. vivax, but this species is virtually

338 absent in the region. Finally, indeed RDTs can provide a false-positive result with patients who had recent

339 malaria episodes. This can be addressed by building a feature into the ConnDx algorithm that patients

340 should be asked whether (s)he experienced malaria episodes in the past 1-2 months and if so, microscopy

341 should be prioritized.

343 All in all, the clinical and economic impact of using RDTs for diagnosing febrile diseases, such as malaria,

344 deserves further attention and studies indicate that this largely depends on the actions undertaken following

345 RDT results. A recent review demonstrated $45 \%$ of patients testing negative for malaria with RDTs still 
346 being prescribed antimalarials by community health workers [38, 39]. The above outlined challenges

347 suggest diagnostics tests for febrile diseases, such as RDTs, should be embedded in a digital infrastructure

348 of logistics and human decision support to raise to the next level of effectiveness and cost reductions. In

349 the future, ConnDx could be deployed for bacterial infections as long as these can be diagnosed by RDTs,

350 leading to better-informed antibiotic prescriptions. This is important in fighting antimicrobial resistance $351[40]$.

353 This study has several strengths and weaknesses. Strength is the important innovation of ConnDx providing

354 reliable, geo-tagged and semi-real-time insights in malaria diagnostic and therapeutic services by private

355 sector providers in a semi-rural setting in Kenya. Kisumu county hosts 94 private providers, which deliver

356 approximately half of all primary healthcare services to its population (the government supplying care

357 through 148 additional providers). With the far majority (1 million) of Kisumu citizens currently connected

358 to M-TIBA [41], ConnDx could in principle rapidly be scaled to all private providers and supplement the

359 governments' DHIS-2 database with valuable real-time private sector information. Another strength is the

360 pioneering nature of this study that was supported by the local health authorities to run in parallel to existing

361 malaria services. This allowed for rapid collection of important data, leading to actionable information for

362 policy makers who demonstrated strong involvement.

364 In terms of weaknesses, this study was an observational study and not a formal clinical trial. Therefore, 365 there are no statistically validated results on (improved) diagnostic performance and (improved) clinical 366 outcomes with respect to malaria. Moreover, in this study there were no special provisions taken for febrile

367 patients who were testing negative for malaria and pertinent consequences with respect to changes in 368 clinical decision making. For example, it was not studied what the effect was of reduced malaria 369 prescription on provider prescription of alternative drugs for fever (in particular, antibiotics) and what were 370 the clinical consequences of such decisions. Furthermore, as the ConnDx process was not yet fully 371 digitalized, several steps were still performed manually, such as linkage of cloud-databases and payout 
372 mechanisms. Therefore, the study did not allow for direct and automated feedback-loops with any of the

373 participating stakeholders (patients, providers, payers, policy makers). For this reason, progress observed

374 with respect to quality improvement or increased cost-efficiency during this pilot, was modest and likely

375 mostly due to the realization of providers that they were being remotely observed by the ConnDx

376 intervention. There were also external factors that influenced the ConnDx pilot, such as civil unrest due to

377 national elections, which hindered uptake of participants due to security issues. There were several strikes

378 of medical staff that put constraints on general malaria service provision. Moreover, M-TIBA is using

379 Safaricom as mobile operator (with a market share of $70 \%$ of Kenya), which became political in Kisumu

380 where most of the population is from other tribal background than the Safaricom ownership, resulting in

381 temporary boycotts of usage of this platform. Finally, it should be kept in mind that ConnDx was

382 implemented in parallel to existing malaria services covered by the NHIF and the MoH. Thus, health

383 providers could in principle benefit by participating in two parallel financing mechanisms, which could

384 potentially create perverse incentives. This would obviously not be the situation when ConnDx is fully

385 integrated into NHIF or any other UHC prepayment mechanism and made a compulsory condition for 386 payouts.

\section{Conclusions}

388 This paper demonstrates the potential of ConnDx for more efficient malaria services at scale. ConnDx links 389 important datasets in (semi) real-time, which previously were in silos and reported irregularly in DHIS-2. 390 This allows for improved efficiencies at all levels of the healthcare system. For clients, the quality of care 391 can improve by avoiding over-prescription of ineffective drugs and by providing the possibility to save and 392 remunerate funds for malaria. Moreover, the linkage of patients' telephone numbers to the platform allows 393 for additional services like malaria information, appointment keeping, adherence support, patient feedback 394 loops to providers on experienced quality of care, individual alarms, early warning systems for geographic 395 malaria hotspots, etc. For providers, better information is given with respect to its diagnosis and treatment 
396 performance versus the National Guidelines, benchmarked and rated against colleagues. Moreover, 397 providers save capitation fees when ConnDx is integrated into NHIF services, avoiding over-prescription 398 of antimalarials. Finally, reputation will be increased due to better-quality care delivered. For payers, 399 ConnDx reduces overhead costs by increasing transparency, supporting healthcare transactions at marginal 400 costs. Funding can be traced to pertinent individual patient cases and vertical malaria funds can be 401 integrated into larger UHC funding pools, covering both public and private sector. For Kenyan policy 402 makers and healthcare managers, ConnDx opens ample opportunities to timely identify weaknesses in 403 service delivery and undertake targeted remedial actions, such as specific training to providers.

404

405 With ConnDx linking cloud-based databases of digital diagnostics data to a digital healthcare exchange 406 platform such as M-TIBA, diagnostic results can target entitlements for malaria treatment directly through 407 the mobile phones of M-TIBA users. This improved financial transparency, combined with marked quality 408 gains through ConnDx presents a valuable proposition for scaling through (inter)national funders, such as 409 the NHIF in Kenya, supported by the GFATM to channel vertical malaria funds through M-TIBA410 facilitated payment platforms and contribute to UHC. ConnDx offers ample opportunities to enable more 411 efficient service delivery for other high-morbidity medical conditions that can be digitally diagnosed, such 412 as cervical cancer and cataract.

413

\section{Abbreviations}

415 ConnDx: Connected Diagnostics; DHIS-2: District Health Information Software; GFATM: Global Fund to 416 Fight AIDS, Tuberculosis and Malaria; IoT: Internet of Things; MoH: Ministry of Health; NHIF: National 417 Hospital Insurance Fund; PMI: President's Malaria Initiative; SSA: sub-Saharan Africa; UHC: Universal 418 Health Coverage; WHO: World Health Organization 


\section{Declarations}

\section{Ethics approval and consent to participate}

421 Written permission was obtained from the Kisumu Department of Health to conduct this study. This

422 paper describes an operational project that was rolled out in Kisumu, Kenya in full coordination with the

423 local Department of Health. The project was not positioned as a research study and therefore no formal

424 ethical clearance was applied for. Rather, we performed this work as an implementation project to

425 improve efficiency of malaria service delivery through innovative digital approaches. As such, this

426 project was approved by the County Chief Office of Health, Dr Dickens Oyango.

428 The Participants of this program were verbally informed about the program by their clinician. When

429 choosing to participate in this program, they subscribed to the M-TIBA digital mobile health platform,

430 which asks clients a question of consent when first registering. For the interviews, verbal and written

431 consent was obtained from the respondents. Confidentiality of research subjects and personnel records

432 was ensured by anonymizing data collection. No individual participant data is disclosed within this

433 manuscript in any form.

434

\section{Consent for publication}

436 Not applicable. 


\section{Availability of data and materials}

439 Due to privacy of patient data, datasets associated with this project are not publicly available. Anonymized 440 data is available from the authors upon request.

442 Competing interests

443 The authors declare no conflicts of interest

444

\section{$445 \quad$ Funding}

446 This study was supported by seed funding from the Joep Lange Institute as well as funding from the

447 Ministry of Foreign Affairs of the Netherlands. The funders of this work had no role further in the study.

448 Their funds were solely used for the implementation of Connected Diagnostics as a digital innovation as 449 part of PharmAccess operations in Kenya.

\section{Authors' contributions}

452 TRW designed the concept, the implementation research study and scientifically supervised the project.

453 EM, AS and EO were responsible for onsite day-to-day operations during the intervention and contributed 454 substantially to data acquisition. MO and MA supported facilitation of the project in health facilities in 455 Kisumu and contributed to collection of data. SD, TRW, LS and SS analyzed and interpreted the data and 456 wrote the first draft manuscript. LS provided complementary essential statistical support. TK provided 457 malaria-specific information for Kisumu, technical assistance to the project and critical review of the paper. 458 DO and LD represented the Kisumu Department of Health, provided policy support, facilitated operations, 
459 reviewed the paper and provided complementary contextual information regarding healthcare access and

460 details on malaria healthcare provision in Kisumu. All authors contributed to writing of this manuscript and

461 have approved the final version of the manuscript for submission.

462

\section{Acknowledgements}

464 The authors would like to express gratitude to all clinicians, nurses, lab technicians, and M-TIBA agents

465 who were involved in the field work of this study, and the respondents who participated in the interviews.

466 We would also like to thank the Kisumu Department of Health for its permission and cooperation to execute

467 this study. Seed funding for this project was generously provided by the Joep Lange Institute.

468 


\section{References}

$470 \quad$ 1. D'Acremont, V., et al., Time to move from presumptive malaria treatment to laboratory-confirmed diagnosis $471 \quad$ and treatment in African children with fever. PLoS Med, 2009. 6(1): p. e252.

472 2. Schroeder, L.F. and T. Amukele, Medical laboratories in sub-Saharan Africa that meet international quality 473 standards. Am J Clin Pathol, 2014. 141(6): p. 791-5.

$4743 . \quad$ Organization, W.H., A report on the misdiagnosis of HIV status. 2014, World Health Organization. p. 31 p. 475 4. WHO, Guidelines for the Treatment of Malaria - 2nd Edition. 2010, World Health Organization. p. 210 p. .

476 5. Yegorov, S., et al., Low prevalence of laboratory-confirmed malaria in clinically diagnosed adult women 477 from the Wakiso district of Uganda. Malar J, 2016. 15(1): p. 555.

478 6. D'Acremont, V., et al., Beyond malaria--causes of fever in outpatient Tanzanian children. N Engl J Med, 479 2014. 370(9): p. 809-17.

4807 7. WH, World Malaria Report 2019 2019, World Health Organization: Geneva.

$481 \quad$ 8. Cibulskis, R.E., et al., Malaria: Global progress 2000 - 2015 and future challenges. Infect Dis Poverty, 2016. $482 \quad 5(1):$ p. 61.

483 9. Patouillard, E., et al., Global investment targets for malaria control and elimination between 2016 and 2030. 484 BMJ Glob Health, 2017. 2(2): p. e000176.

485 10. President's Malaria Initiative (PMI). 201931 December 2019]; Available from: https://www.pmi.gov/.

486 11. The Global Fund. 201931 December 2019 ]; Available from: https://www.theglobalfund.org/en/.

487 12. Wang, J., et al., Preparedness is essential for malaria-endemic regions during the COVID-19 pandemic. The $488 \quad$ Lancet 2020.

489 13. Jones, S.A., et al., 'Women and babies are dying but not of Ebola': the effect of the Ebola virus epidemic on 490 the availability, uptake and outcomes of maternal and newborn health services in Sierra Leone. BMJ Glob $491 \quad$ Health, 2016. 1(3): p. e000065.

492 14. Afrane, Y.A., et al., Utility of health facility-based malaria data for malaria surveillance. PLoS One, 2013. $493 \quad$ 8(2): p. e54305.

494 15. Oladosu, O.O. and W.A. Oyibo, Overdiagnosis and Overtreatment of Malaria in Children That Presented 495 with Fever in Lagos, Nigeria. ISRN Infectious Diseases, 2013.

496 16. Hume, J.C., et al., Household cost of malaria overdiagnosis in rural Mozambique. Malar J, 2008.7 : p. 33. 
497 17. Were, V., et al., Socioeconomic health inequality in malaria indicators in rural western Kenya: evidence 498 from a household malaria survey on burden and care-seeking behaviour. Malar J, 2018. 17(1): p. 166.

499 18. Miorandi, D., S.D.P. Sicari, F., and C. I., Internet of things: Vision, applications and research challenges. $500 \quad$ Ad Hoc Networks, 2012. 10: p. 1497-1516.

501 19. Chutel, L. Africa's largest phone network is expanding its dominance with its own "smart feature phone"

502201831 December 2019]; Available from: https://qz.com/africa/1464244/africas-largest-phone-network-is503 expanding-its-dominance-with-its-own-smart-feature-phone/.

504 20. Databank, W. World Development Indicators - Mobile Cellular Subscriptions 2017201731 December 2019

505 ]; Available from: https://databank.worldbank.org/data/source/world-development-indicators.

506 21. Safaricom. Safaricom - MPESA. $2019 \quad 31$ December 2019]; Available from: 507 https://www.safaricom.co.ke/personal/m-pesa.

508 22. M-TIBA Dashboard. 201931 December 2019]; Available from: http://pharmaccess.m-tiba.org/home.

509 23. Foundation, P. HealthConnect. 31 December 2019]; Available from: https://www.healthconnectme.org/.

510 24. Smith, S., et al., Connected diagnostics: linking digital rapid diagnostic tests and mobile health wallets to 511 diagnose and treat brucellosis in Samburu, Kenya. BMC Med Inform Decis Mak, 2019. 19(1): p. 139.

512 25. Abdelhady R, A.K., Elhussein A., Prevelance of brucellosis among febrile negative malaria patients by PCR 513 in Northern Kordofan State, Sudan. . Clin Microbiol, 2017. 6(293): p. 2.

514 26. KNBS, 2019 Kenya Population and Housing Census Volume I: Population by County and Sub-County. 2019, $515 \quad$ Kenya National Bureau of Statistics.

$51627 . \quad$ Jenkins, R., et al., Prevalence of malaria parasites in adults and its determinants in malaria endemic area of 517 Kisumu County, Kenya. Malar J, 2015. 14: p. 263.

518 28. Kisumu County - Health at a glance. $2015 \quad 31$ December 2019 ]; Available from: 519 https://www.healthpolicyproject.com/pubs/291/Kisumu\%20County-FINAL.pdf.

520 29. WorldBank. 31 December 2019]; Available from: https://microdata.worldbank.org/index.php/catalog/2660.

521 30. Kisumu Monthly Climate Averages - Rainfall and Rain Days [cited 2020 February, 6]; Available from: 522 https://www.worldweatheronline.com/kisumu-weather-averages/nyanza/ke.aspx.

523 31. Kenya Malaria Indicator Survey 2015 2016, ICF international, Kenya National Bureau of Statistics Nairobi, $524 \quad$ Maryland 
525 32. Patel, A., et al., Quality of generic medicines in South Africa: perceptions versus reality - a qualitative study. $526 \quad$ BMC Health Serv Res, 2012. 12: p. 297.

527 33. Fadare, J.O., et al., The prescribing of generic medicines in Nigeria: knowledge, perceptions and attitudes of 528 physicians. Expert Rev Pharmacoecon Outcomes Res, 2016. 16(5): p. 639-650.

529 34. Kalogriopoulos, N.A., et al., Electronic medical record systems for developing countries: review. Conf Proc 530 IEEE Eng Med Biol Soc, 2009. 2009: p. 1730-3.

531 35. Ministry of Public Health and Sanitation, M.o.M.S., NATIONAL GUIDELINES FOR THE DIAGNOSIS, TREATMENT AND PREVENTION OF MALARIA IN KENYA. 2010.

533 36. de Oliveira, A.M., et al., Performance of malaria rapid diagnostic tests as part of routine malaria case 534 management in Kenya. Am J Trop Med Hyg, 2009. 80(3): p. 470-4.

535 37. Proux, S., et al., Paracheck-Pf: a new, inexpensive and reliable rapid test for P. falciparum malaria. Trop 536 Med Int Health, 2001. 6(2): p. 99-101.

537 38. Chandler, C.I., C.J. Whitty, and E.K. Ansah, How can malaria rapid diagnostic tests achieve their potential? 538 A qualitative study of a trial at health facilities in Ghana. Malar J, 2010. 9: p. 95.

539 39. Ruizendaal, E., et al., Success or failure of critical steps in community case management of malaria with 540 rapid diagnostic tests: a systematic review. Malar J, 2014. 13: p. 229.

541 40. Legese A. Mekuria, T.F.d.W., Nicole Spieker,Ramona Koech,Robert Nyarango,Stanley Ndwiga,Christine J. Fenenga,Alice Ogink,Constance Schultsz,Anja van't Hoog, Analyzing data from the digital healthcare exchange platform for surveillance of antibiotic prescriptions in primary care in urban Kenya: A mixedmethods study. Plos One 2019.

41. Massive UHC Registration Drive to get underway in Kisumu County Kenya. 201831 December 2019 ]; 


\section{Figures}

\section{Figure 1.}

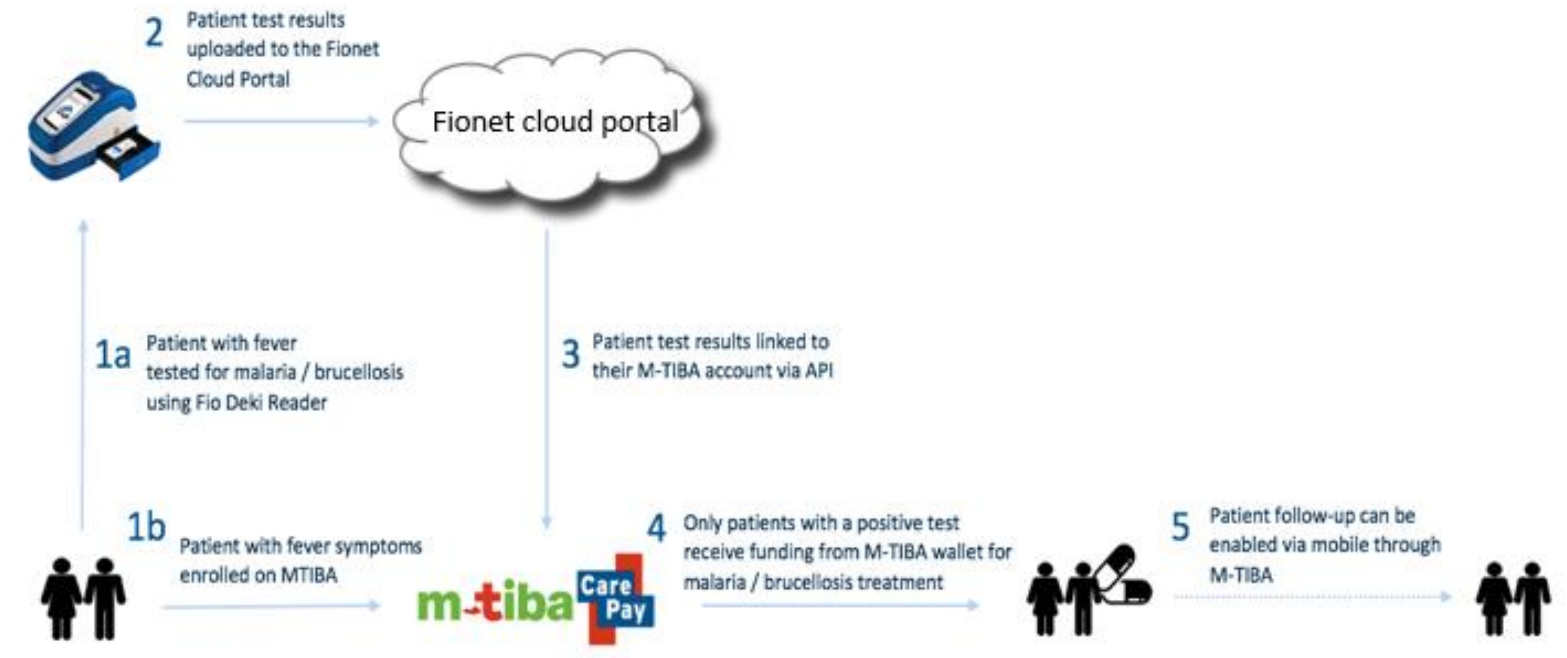

Figure 1. Schematic illustration ConnDx process. Step 1) Patient with fever symptoms is tested for malaria and enrolled on M-TIBA. 2) Test results of patient are uploaded to the cloud portal of Fionet. 3) The test results are also linked to the M-TIBA account of the patient. 4) Patients with a positive test receive funding for treatment. 5) Patient follow-up can be enabled via mobile through M-TIBA.

\section{Figure 2.}

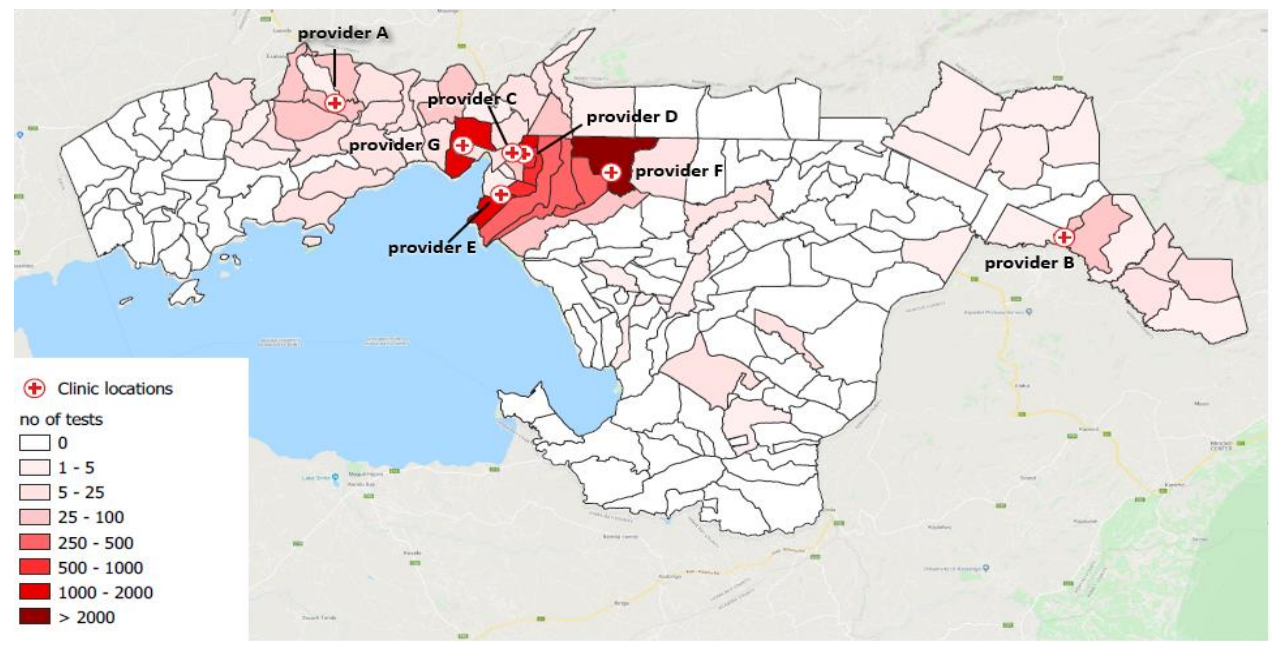

Figure 2. Location of the participating providers A-G in Kisumu County and hotspots of malaria tests. Data used to construct this map is gathered during this intervention.

\section{Figure 3.}




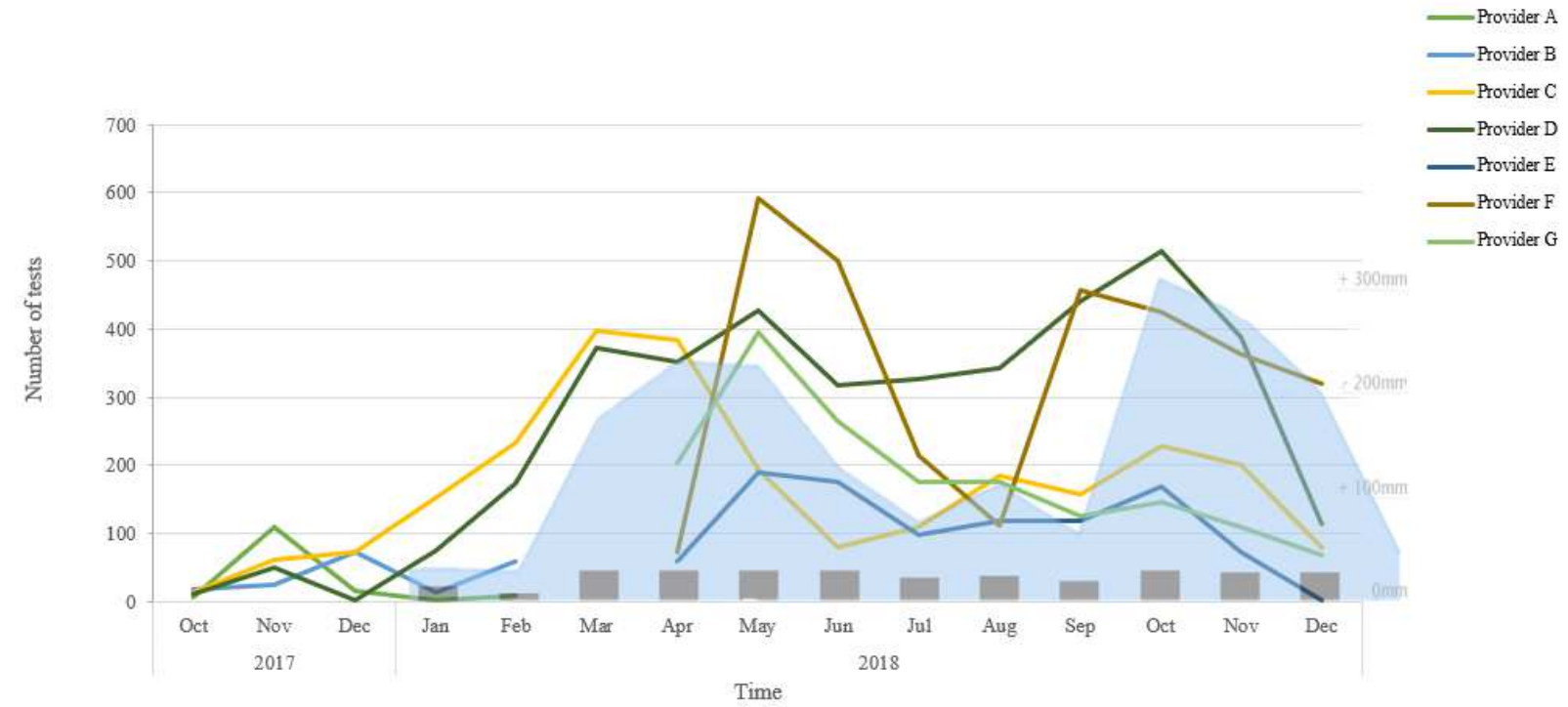

Figure 3. Participation dynamics providers $A-G$ during the pilot of ConnDx in Kisumu. Number of transactions (tests) per provider from October 2017 - December 2018 derived from FIO database. Monthly rainfall in 2018 is indicated grey bars per months and in the light blue graph. Derived from worldweatheronline.com

\section{Figure 4.}

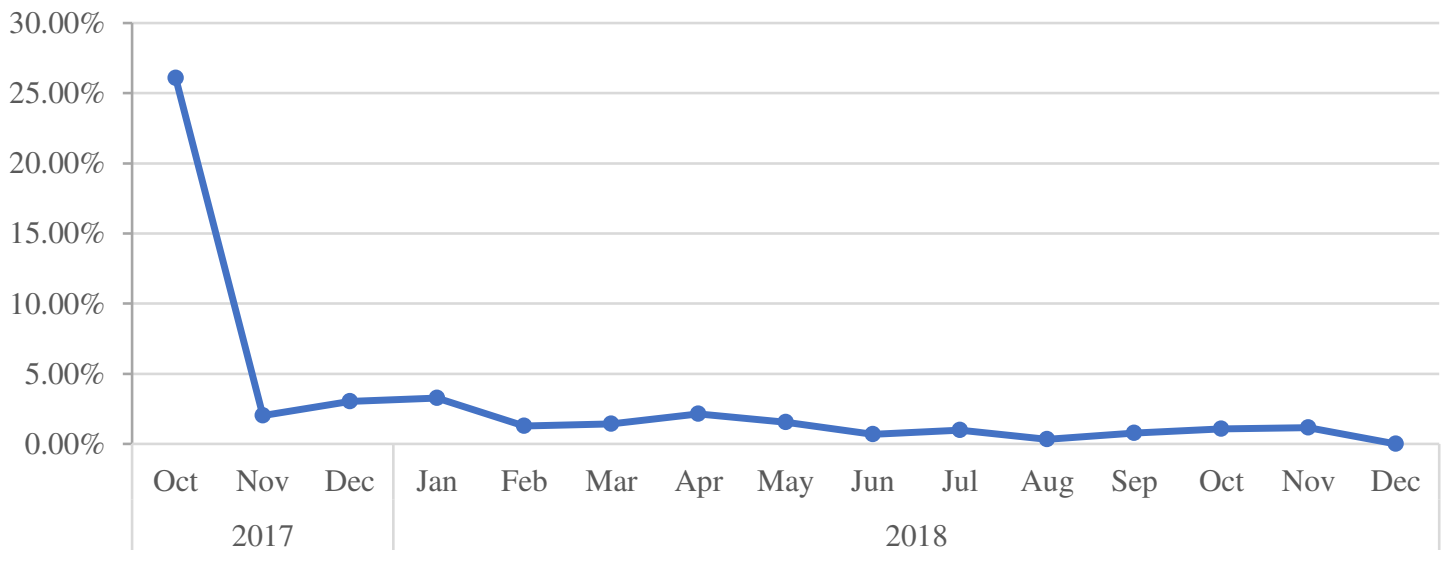

Figure 4a. Overall percentage of RDTs with errors, from October 2017 - December 2018 


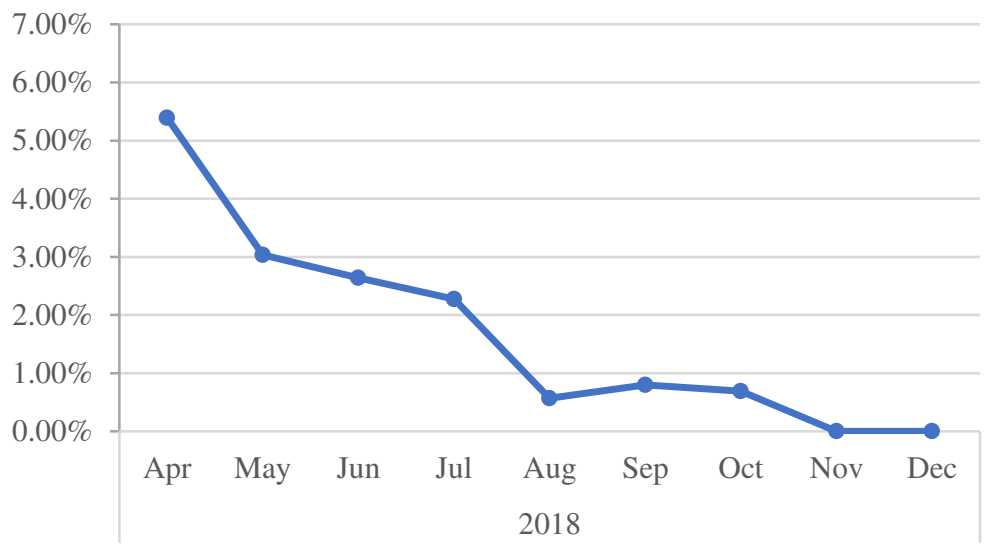

Figure 4b. Provider $G$ percentage of RDTs with errors, from April 2018 - December 2018

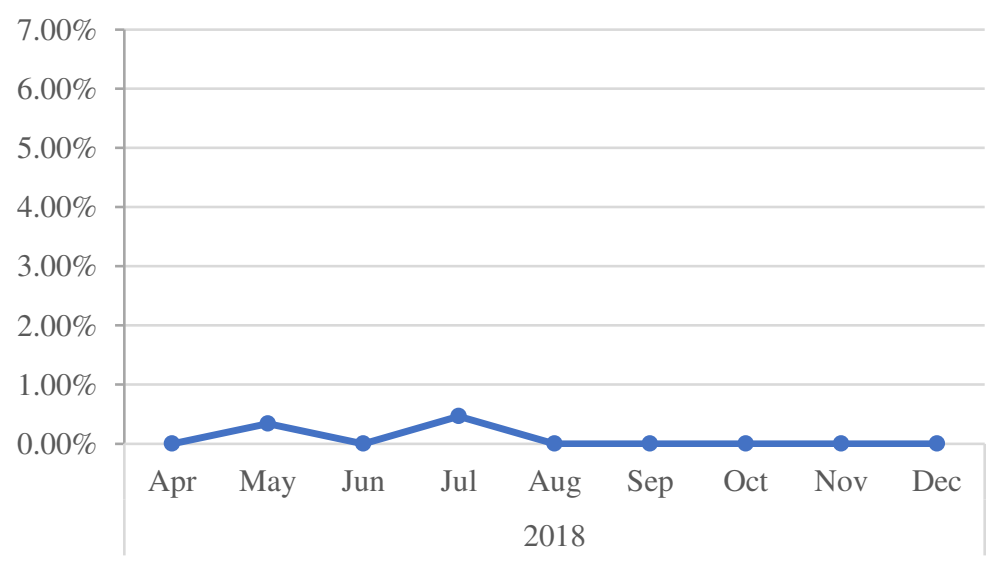

Figure 4c. Provider F percentage of RDTs with errors, from April 2018 - December 2018

\section{Figure 5.}

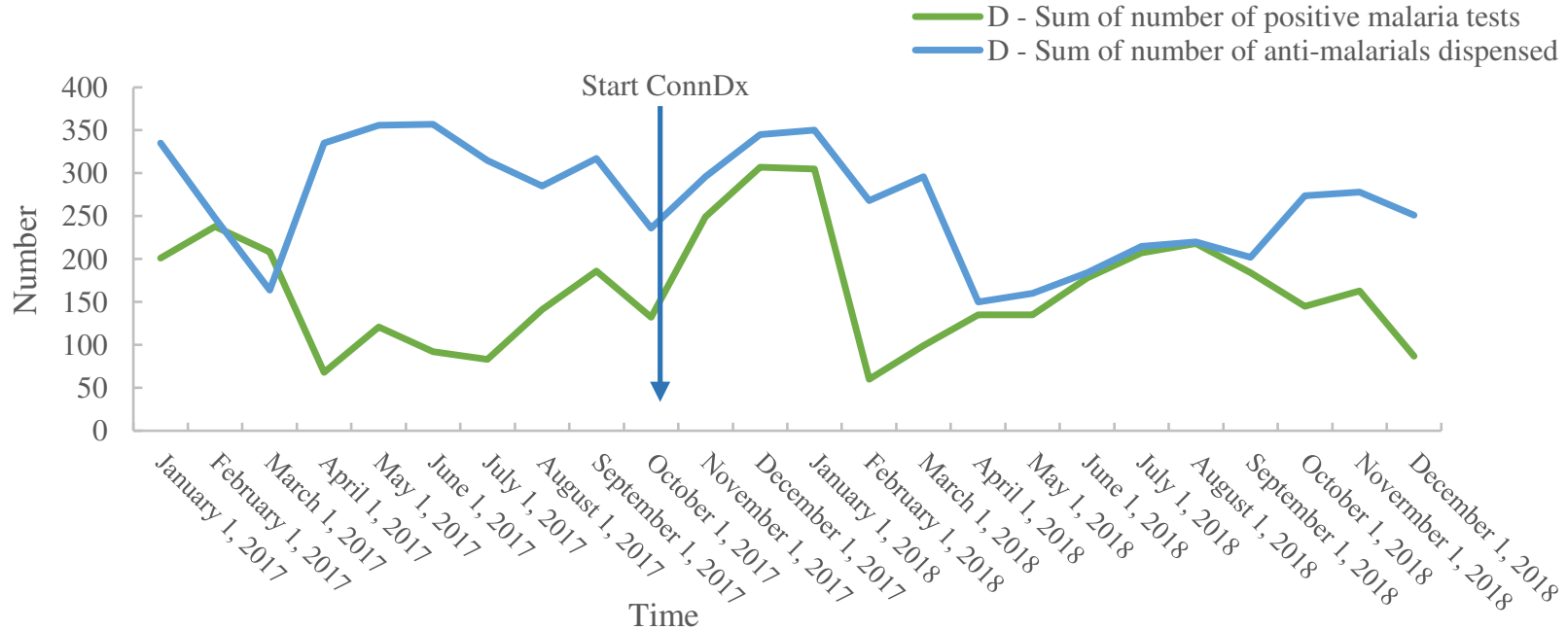

Figure 5. Number of positive malaria tests compared with number of antimalarials prescriptions dispensed within provider $D$ throughout the project and the 9 months prior to implementation. 


\section{Figure 6.}

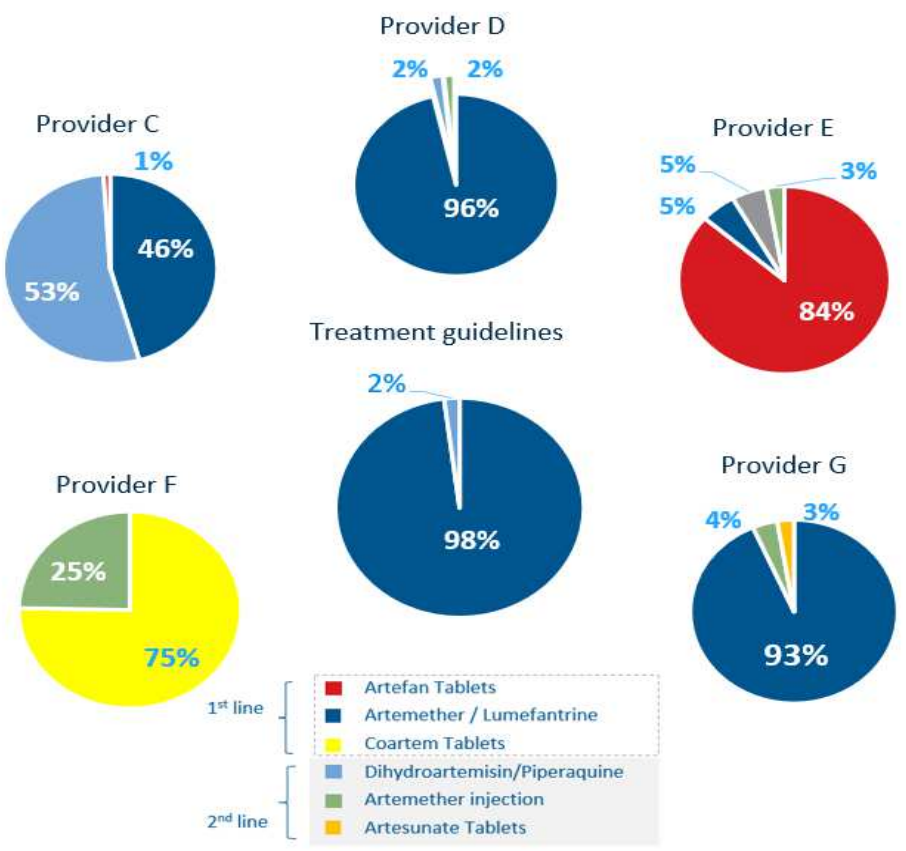

Figure 6. Type of antimalarials dispensed during ConnDx pilot per provider, divided in $1^{\text {st }}$ versus $2^{\text {nd }}$ line 


\section{Figures}

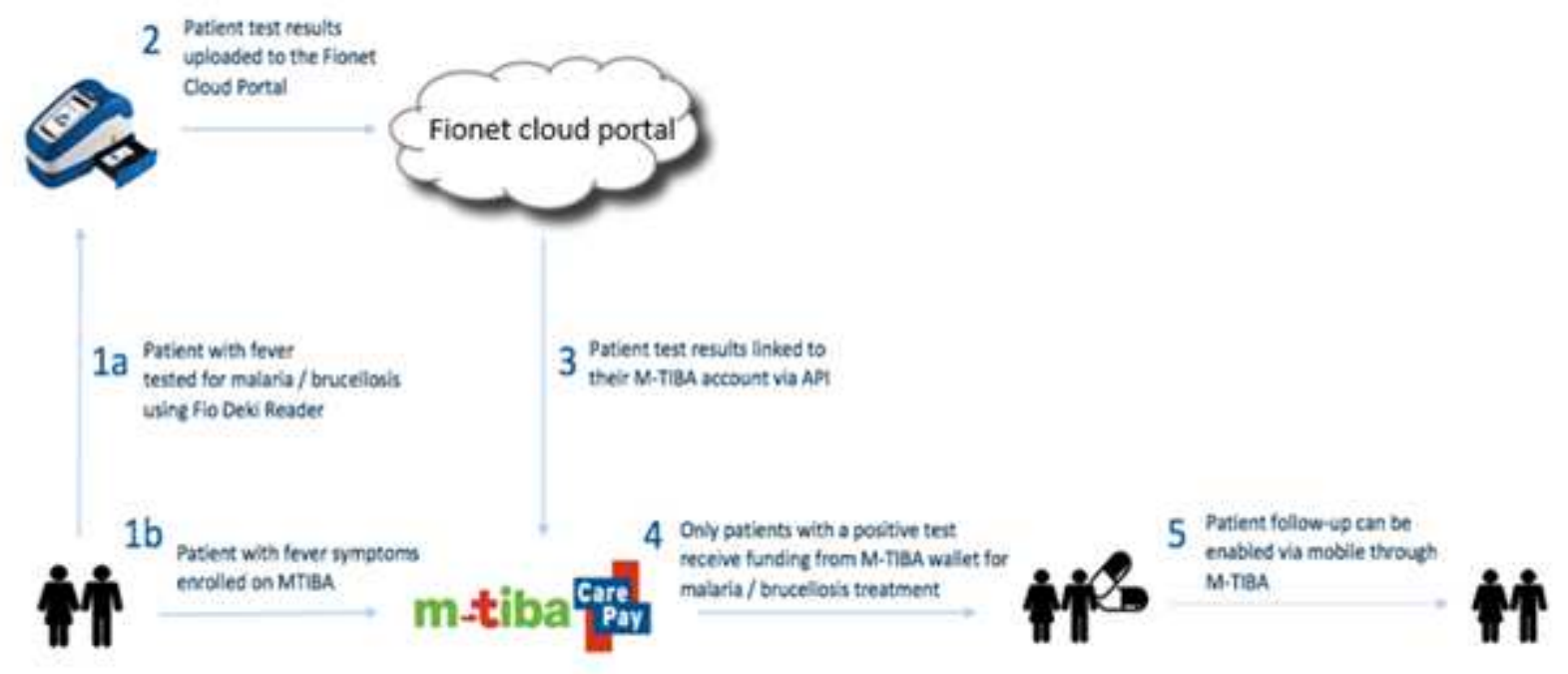

Figure 1

Schematic illustration ConnDx process. Step 1) Patient with fever symptoms is tested for malaria and enrolled on M-TIBA. 2) Test results of patient are uploaded to the cloud portal of Fionet. 3) The test results are also linked to the M-TIBA account of the patient. 4) Patients with a positive test receive funding for treatment. 5) Patient follow-up can be enabled via mobile through M-TIBA.

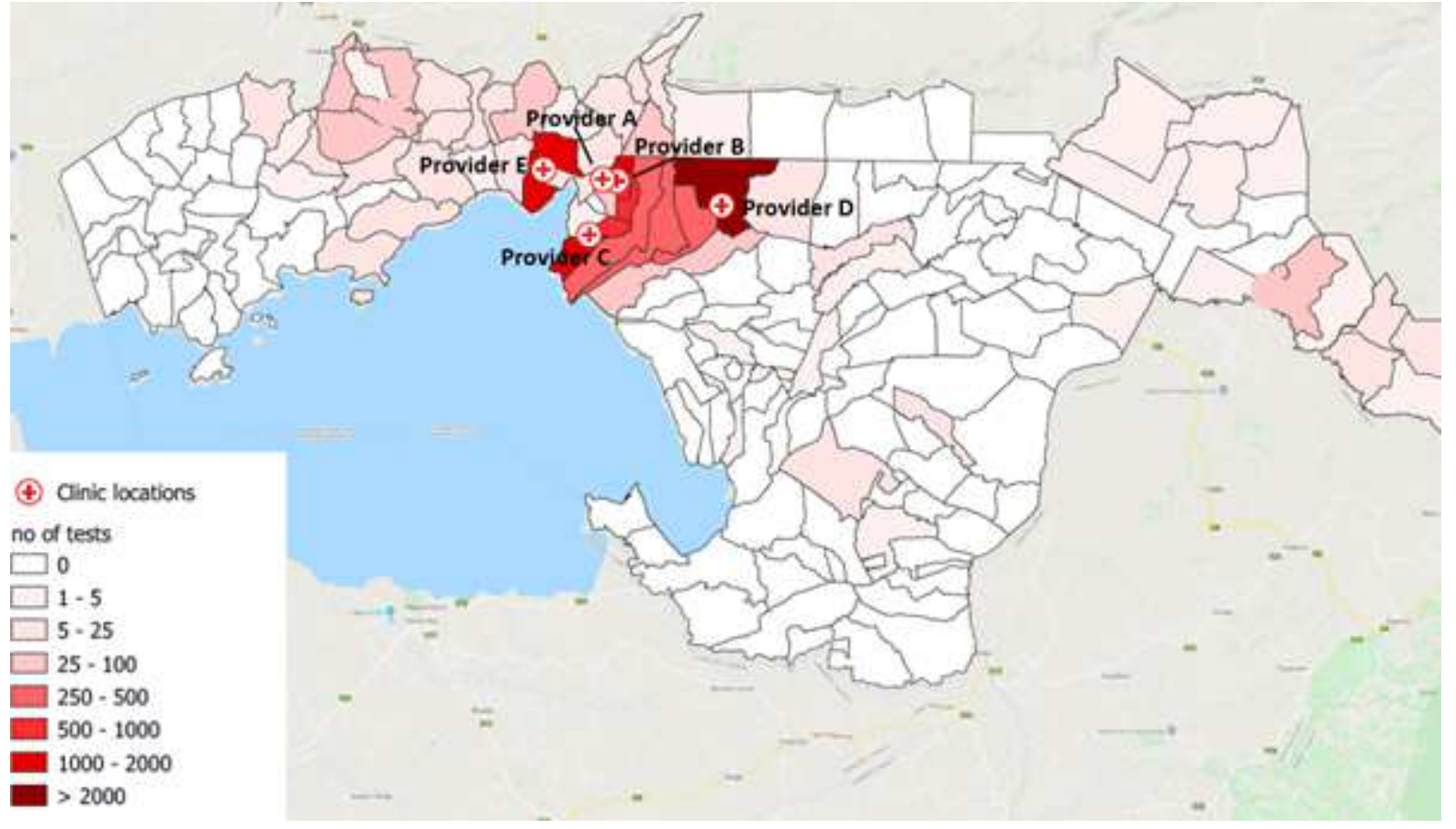

Figure 2

Location of the participating providers A-E in Kisumu County and hotspots of malaria tests. Data used to construct this map is gathered during this intervention. Note: The designations employed and the presentation of the material on this map do not imply the expression of any opinion whatsoever on the 
part of Research Square concerning the legal status of any country, territory, city or area or of its authorities, or concerning the delimitation of its frontiers or boundaries. This map has been provided by the authors.

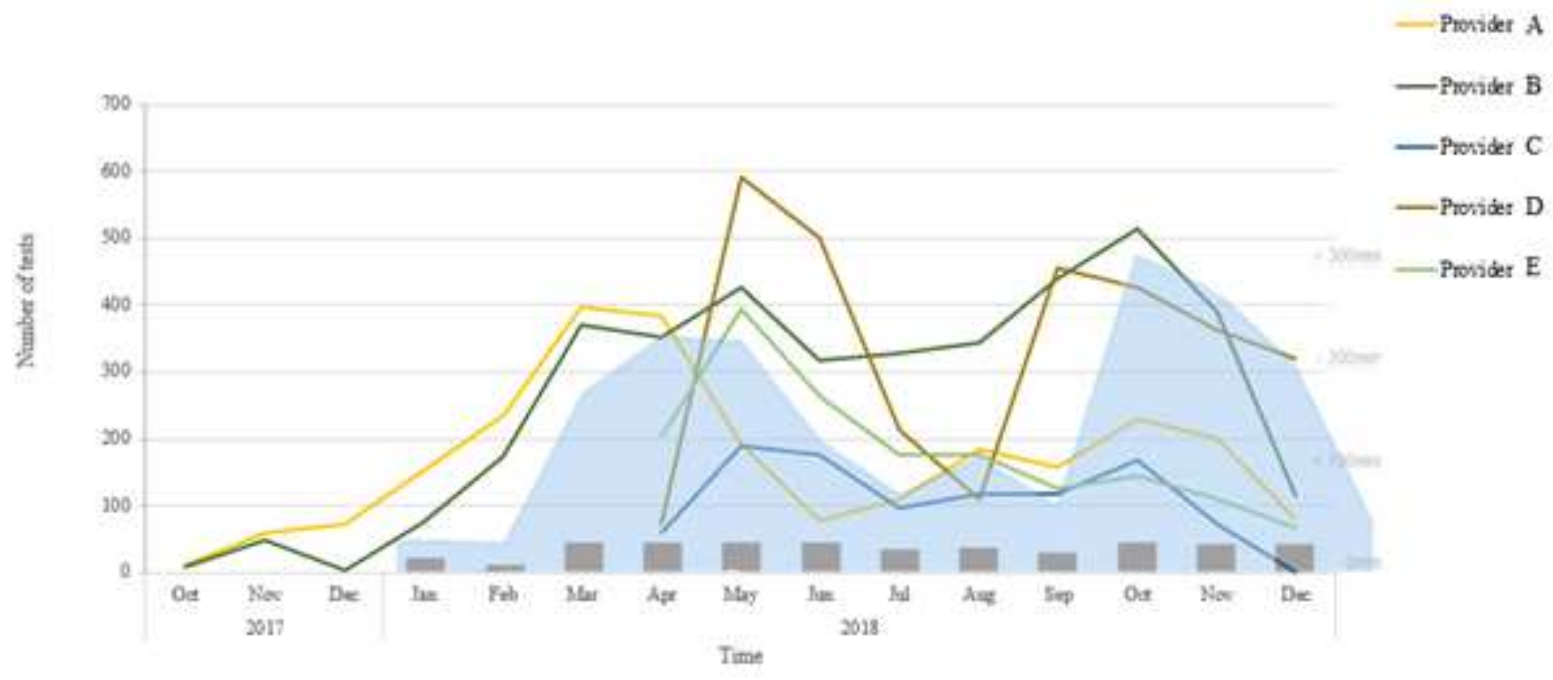

\section{Figure 3}

Participation dynamics providers A - E during the pilot of ConnDx in Kisumu. Number of transactions (tests) per provider from October 2017 - December 2018 derived from FIO database. Monthly rainfall in 2018 is indicated grey bars per months and in the light blue graph. Derived from worldweatheronline.com 
a.

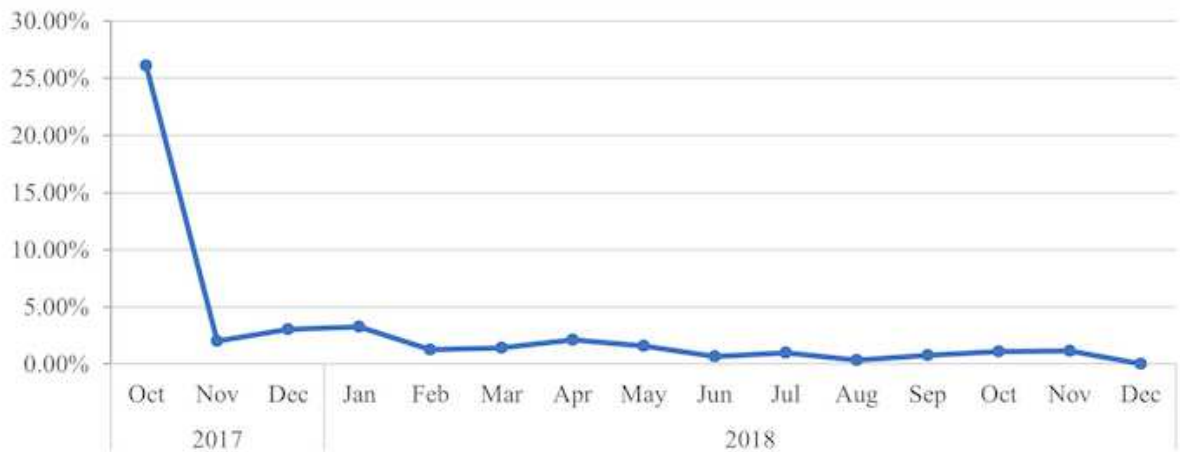

b.

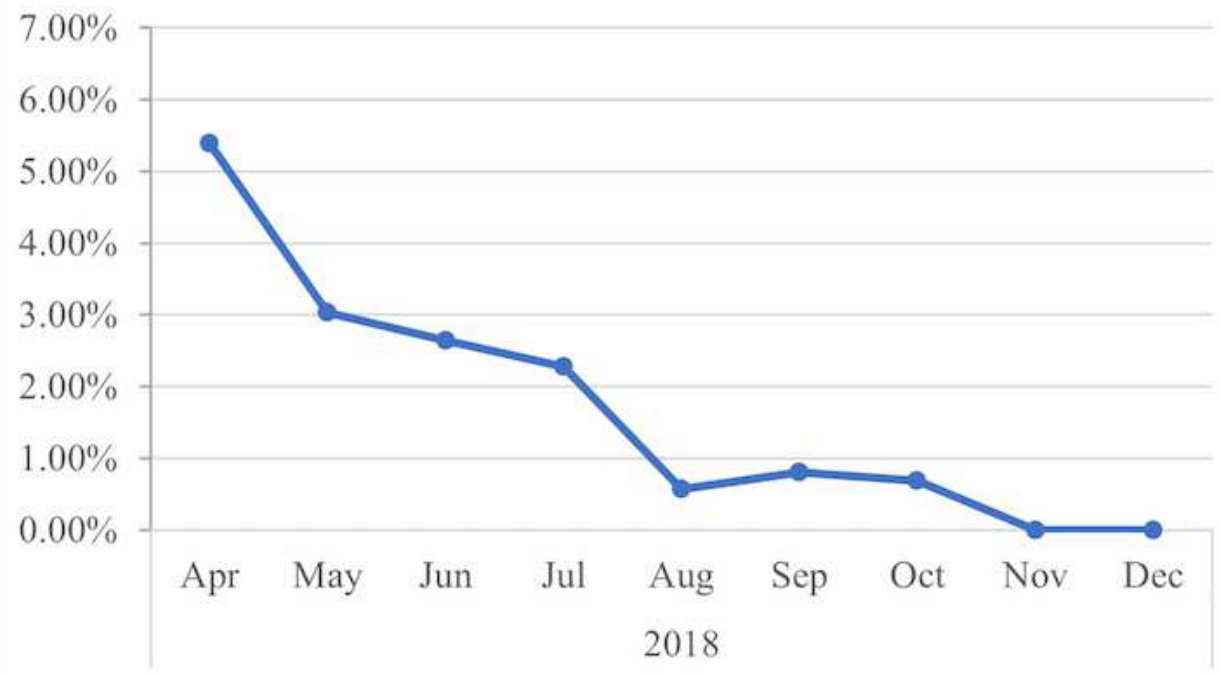

c.

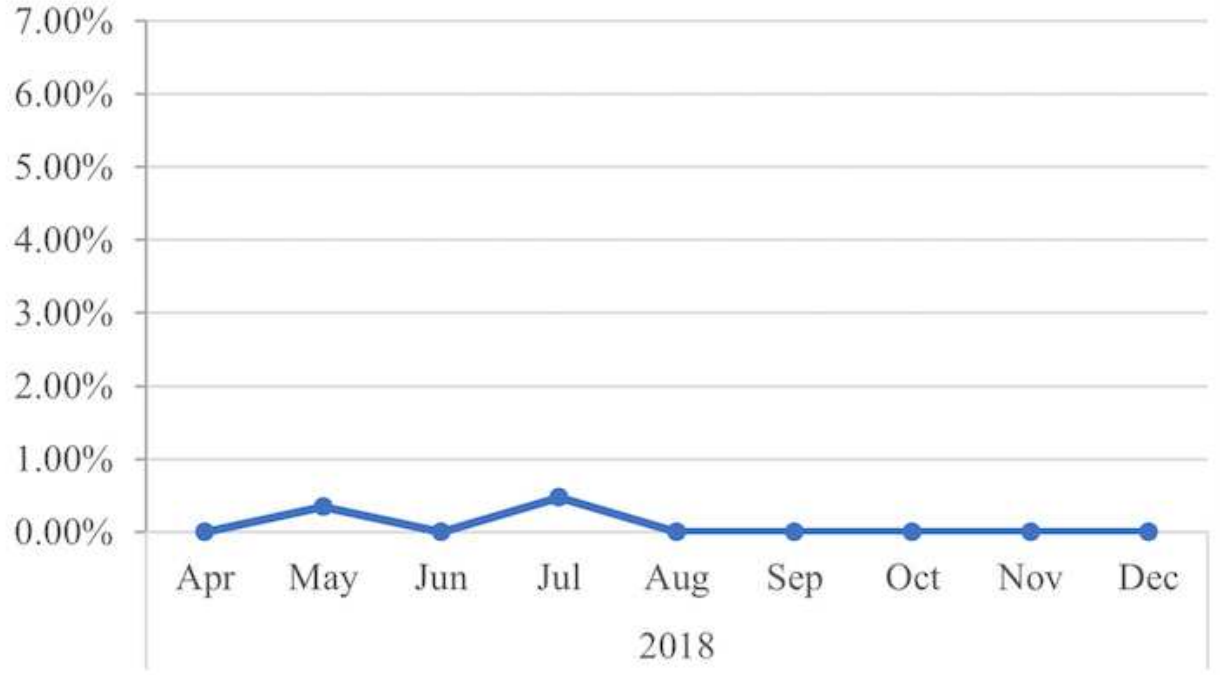

Figure 4

a. Overall percentage of RDTs with errors, from October 2017 - December $2018 \mathrm{~b}$. Provider E percentage of RDTs with errors, from April 2018 - December 2018 c. Provider D percentage of RDTs with errors, from April 2018 - December 2018 


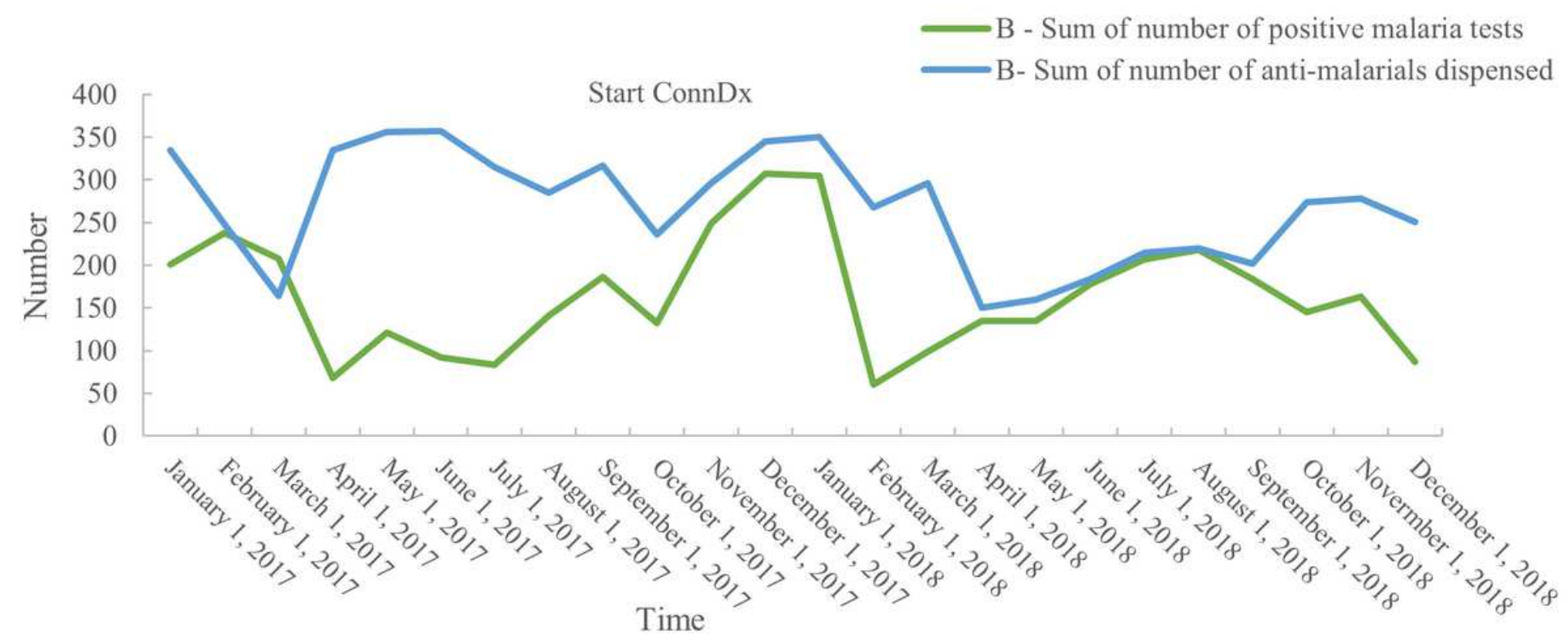

\section{Figure 5}

Number of positive malaria tests compared with number of antimalarials prescriptions dispensed within provider $B$ throughout the project and the 9 months prior to implementation.

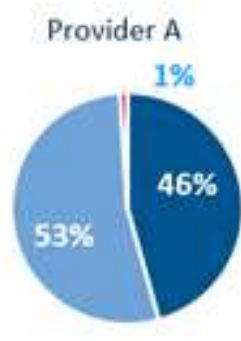

Provider B
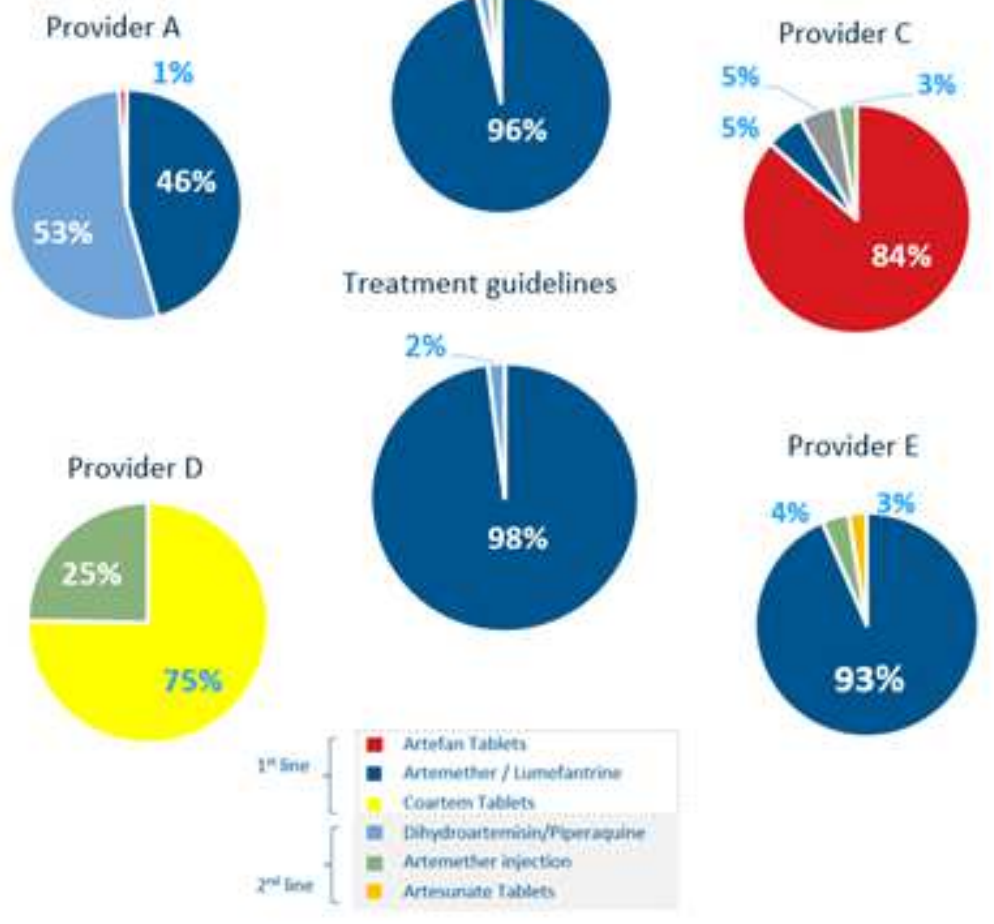

Provider E

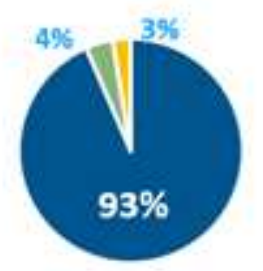

\section{Figure 6}

Type of antimalarials dispensed during ConnDx pilot per provider, divided in 1 st versus 2 nd line 